\title{
Eencephaloduroarteriosynangiosis for hemorrhagic moyamoya disease: long-term outcome of a consecutive series of 95 adult patients from a single center
}

\section{Lian Duan.}

The people's Liberation Army 307 Hospital, neurosurgery, Beijing, China.

To investigate long term outcomes after encephaloduroarteriosynangiosis (EDAS) for the treatment of hemorrhagic moyamoya disease (MMD) and identify the risk factors for recurrent hemorrhages. We retrospectively reviewed 95 patients with haemorrhagic MMD who were treated with EDAS at 307 Hospital PLA. Clinical features, angiographic findings, and clinical outcomes were investigated. Rebleeding incidences were compared between anterior or posterior hemorrhagic sites. Kaplan-Meier survival analysis and Cox proportional hazard regression models were used to estimate rebleeding risks post EDAS. The average age at symptom onset was 37.1 years for adult patients. The ratio of female to male patients was 1.16:1. In 61 of 95 hemorrhagic hemispheres $(64.2 \%)$, the anterior choroidal artery or posterior communicating artery (AChA-PCoA) was extremely dilated, with extensive branches beyond the choroidal fissure, which only occurred in 28 of 86 nonhemorrhagic hemispheres (32.6\%). 57 incidences were classifed as anterior hemorrhages and 38 as posterior. Sixteen of 95 patients $(16.8 \%)$ suffered cerebral rebleeding after a median follow-up time of 8.5 years. The annual rebleeding rate was $2.2 \% /$ person/year. The incidence rate was higher for the posterior group than for the anterior group, however, this difference was not statistically significant $(p>0.05)$. Cox regression analysis revealed that the age of symptom onset $(P=0.028)$ was a predictor of rebleeding strokes. EDAS proves beneficial for patients with hemorrhagic MMD. Dilation of the AChA-PCoA is associated with the initial

\begin{tabular}{rrccc} 
& Variables & Anterior(n=57) & Posterior $(\mathrm{n}=38)$ & Pvalue \\
\cline { 2 - 5 } & Mean age \pm SD, & $36.5 \pm 6.9$ & $37.9 \pm 9.6$ & 0.398 \\
Female (\%) & $26(45.6)$ & $25(65.8)$ & 0.053 \\
Stroke risk factors & & & \\
Hypertension (\%) & $10(17.5)$ & $6(15.8)$ & 0.823 \\
& Diabetes mellitus (\%) & $4(7.0)$ & $1(2.6)$ & 0.348 \\
Hyperlipidemia (\%) & $2(3.5)$ & 0 & 0.243 \\
& $7(12.3)$ & $4(10.5)$ & 0.793 \\
& Smoking or drinking (\%) & & \\
& Types of haemorrhage (\%) & $25(43.9)$ & $15(39.5)$ & 0.929 \\
ICH & IVH & $21(36.8)$ & $14(36.8)$ & \\
& SAH & $7(12.3)$ & $5(13.2)$ & \\
& $4(7.0)$ & $4(10.5)$ & 0.173 \\
& IVH with ICH & $13(22.8)$ & $7(14.2)$ & 0.344 \\
PCI (\%) & & & \\
& Follow-up events (\%) & & & \\
Rebleeding & $8(14.0)$ & $1(21.1)$ & 0.748 \\
\hline
\end{tabular}

Figure 1. Kaplan-Meier estimates of rebleeding incidences after surgery.
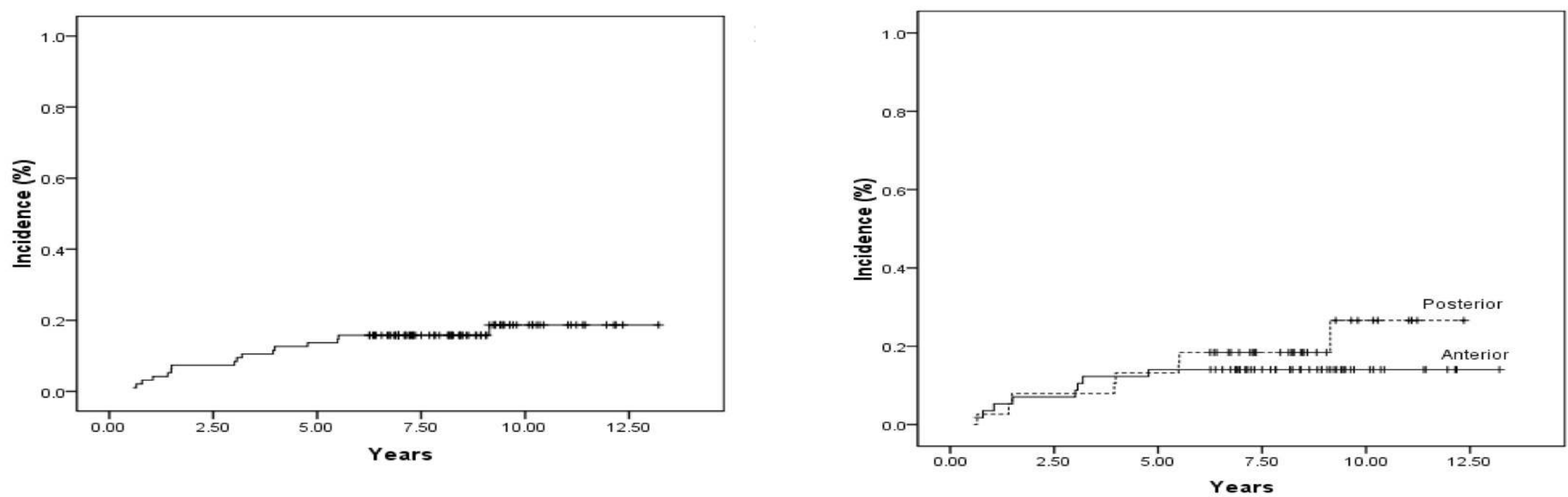